\title{
Joint Routing and Resource Allocation for Software Defined Mobile Networks
}

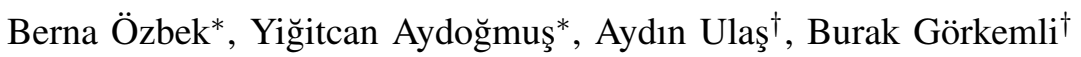 \\ *Electrical and Electronics Engineering Department, Izmir Institute of Technology, Izmir, Turkey \\ ${ }^{\dagger}$ ARGELA A.Ş., İstanbul,Turkey \\ E-mail:\{bernaozbek@iyte.edu.tr\}
}

\begin{abstract}
The need for software defined mobile networking (SDMN) increases to manage the complexity in communication networks for the fifth generation mobile networks and beyond, with increasing diverse demand on data traffic in wireless environments. The separation of the data and control planes offers flexibility in future networks with SDMN by taking into account to the wireless access problem in complex radio environments. In this paper, we propose an efficient joint routing and resource allocation algorithm to minimize the cost based on power consumption while satisfying both throughput and delay requirement of the flows under a given capacity of links in night time traffic through SDMN. The power consumption is determined based on the number of active OpenFlow switches and active ports in the network. In the proposed joint algorithm, we put the selected network components to sleep mode in order to reduce overall network power consumption. The performances of the proposed algorithm are illustrated under different throughput constraints in various network topologies and scenarios in night-time traffic.
\end{abstract}

Index Terms-Software defined mobile network, routing, resource allocation, power efficiency

\section{INTRODUCTION}

There is a strong need to manage the traffic carried by networks since the traffic diversity and volume increase with growing popularity of mobile applications. Software defined networking (SDN) can simplify network management while enabling new services by employing traffic management including routing whose goal is to maximize a given utility function while satisfying capacity requirements. Another key concept to meet up huge data traffic is cloud-based radio access networks. By integrating cloud services to radio access networks, operators will make use of network functions virtualization which allows to host different virtualized functions on a common hardware platform.

SDN can reduce network complexity, hence it is suitable for sophisticated environments such as a mobile networks [1]. Traffic management have received a great deal of attention within the OpenFlow/SDN community [2]. One of the goals of traffic management is to decide how to route traffic in a network in order to balance several objectives such as maximizing throughput, balancing link utilization, minimizing power consumption, controlling the bandwidth allocated to competing flows and minimizing latency. There are several traffic management strategies examined for SDNs.

In [3], an energy efficient routing scheme with traffic aggregation for each flow to decrease the number of active network devices while maintaining acceptable performance was presented. In this work, how to optimize power management globally at network level was considered by re-routing traffic through different paths to adjust the load of links when the network is relatively idle. In [4], the energy saving mechanism has been presented by turning on a minimal amount of network devices to carry the traffic, instead of powering on all switches at all time by formulating as an Mixedinteger linear programming (MILP) problem. In [5], an energy efficient controller association algorithm was given by switching off the maximum number of links by taking into account several constraints on delay, link and controller load. In our previous work in [6], an energy efficient routing algorithm to decrease the number of active OpenFlow switches in the network without taking into account the delay constraints have been presented. In [7], an efficient and effective video transmission scheme for enhancement of video delivery was examined by using genetic algorithm based SDN routing algorithm.

SDN was integrated to the very dense heterogeneous wireless networks. However, there are some limitations in dense networks due to constraints such as radio access network bottlenecks, control overhead, and high operational costs [8] [9]. In [10], a heterogeneous scenario, where machine to machine and long term evolution (LTE) interoperation has been examined in SDN to make LTE cellular network much simpler in order to manage and reconfigure, by introducing new features and interoperating with other technologies. Indeed, SDN is a key issue also in cellular networks [11].

One of the promising solutions is based on integrating cloud services to radio access networks, called Cloud Radio Access Networks (C-RAN). It is a centralized, backwards compatible and cloud computing-based architecture for radio access networks for future communication standards by increasing network capacity and coverage. C-RAN is a centralized and cloud computing- 
based architecture for radio access networks [12]. CRAN architecture uses combination of virtualization, centralization and coordination techniques. In [13], a joint power and subchannel allocation algorithm for mobile network virtualization to achieve required quality of services was presented for orthogonal frequency division multiple access (OFDMA) based systems with multiple virtual network operators.

In this paper, we consider a software defined mobile network (SDMN) which employs both C-RAN and SDN. For SDMN, we propose a joint routing and resource allocation algorithm to reduce the power consumption based on the number of active OpenFlow switches and active ports in the network while satisfying both throughput and delay requirements of flows. The proposed algorithm achieves the data rate requirements through C-RAN and provides these information to SDN in order to shut down the unnecessary switches and ports to improve energy efficiency. The performances of the proposed algorithm for different throughput constraints in various network topologies and scenarios are demonstrated in night-time traffic in SDMN. Section II gives the considered network model. Section III proposes a joint routing and resource allocation algorithm in detail. Section IV and Section V give the performance results and the conclusion, respectively.

\section{NETWORK MODEL}

We consider a network model given in Figure 1 including joint SDN and C-RAN.

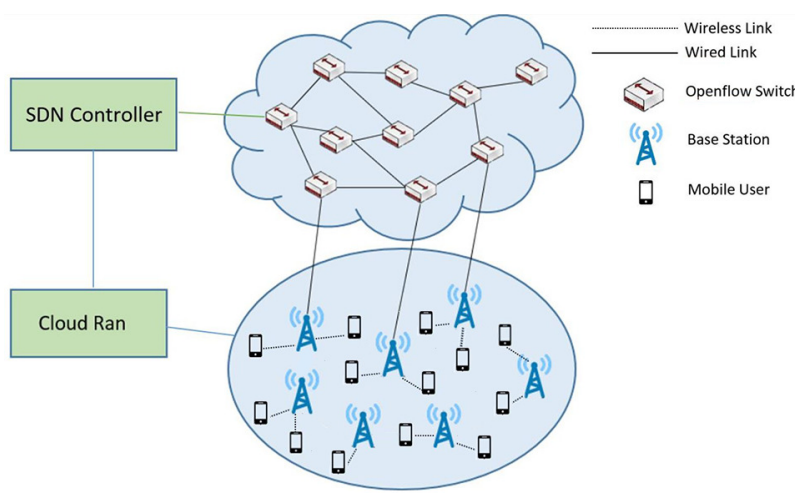

Fig. 1: The network model

The network is modelled as a graph $G<\mathbb{V}, \mathbb{Z}>$ where $\mathbb{V}$ is the set of switches and $\mathbb{Z}$ is the set of possible links between the switches through available ports. $N_{u, t}$ links can be established between node $u$ and node $t$ proportional to the number of ports in the nodes. Each link $\left(u_{y}, t_{y}\right) \in \mathbb{Z}$ has a capacity $C_{\left(u_{y}, t_{y}\right)}$ where $y=1, \ldots, N_{u, t}$. The capacity of each link is fixed and it is adjusted according to the number of available ports in the switches and the maximum available capacity, $C_{(u, t)}$. Let $\mathbb{F}$ be the set of $K$ flows. Each flow $f_{k} \in \mathbb{F}$ has several parameters, source, $\mathrm{s}_{f_{k}}$, destination, $\mathrm{d}_{f_{k}}$, throughput requirement, $R_{f_{k}}$ and delay requirement $d_{f_{k}}$.

In this work, the aim of our solution is to find all routes $\mathbf{p}=\left\{\mathrm{p}_{f_{1}}, \mathrm{p}_{f_{2}}, \ldots, \mathrm{p}_{f_{K}}\right\}$ where $\mathrm{p}_{f_{k}}$ is the set of routes composed of active links from source to destination belonging to the flow $f_{k}$. While finding the routes $\mathbf{p}$ that satisfy throughput requirements of all flows which are determined based on availability of wireless links and users' data rate requests, we reduce the power consumption based on the number of active switches and the number of active links in the network. Let $x_{v} \in\{0,1\}$ indicates whether the switch $v$ is active or not. If the switch $v$ is used through the links selected for the route $\mathbf{p}$, then its indicator, $x_{v}$, becomes 1 . Let $x_{z} \in\{0,1\}$ indicate whether the link $z$ is active or not. If the link $z$ is used for the route $\mathbf{p}$, then its indicator, $x_{z}$, becomes 1 .

The total power consumption in the network is determined by

$$
P(\mathbf{p})=\sum_{v \in \mathbb{V}} x_{v}(\mathbf{p}) P_{\text {switch }}+\sum_{z \in \mathbb{Z}} x_{z}(\mathbf{p}) P_{\text {port }}
$$

where $P_{\text {switch }}$ and $P_{\text {port }}$ are the power consumption at the switch and at the port, respectively.

In order to design a power efficient network, the optimization problem is defined as,

$$
\min P(\mathbf{p})
$$

subject to

$$
\begin{gathered}
R_{f_{k}} \leq C_{f_{k}}^{w} \quad \forall f_{k} \\
\sum_{y=1}^{N_{u, t}} C_{\left(u_{y}, t_{y}\right)} \leq C_{(u, t)} \quad \forall u, \forall t \\
\mathrm{~d}_{f_{k}} \leq D^{\max } \quad \forall f_{k}
\end{gathered}
$$

where $D^{\max }$ is the maximum acceptable delay and $C_{f_{k}}^{w}$ represents the maximum data rate through flows and is determined by taking into account wireless links availability and users data rate demand.

Eq. (3) states that the link capacity must be equal to or lower than throughput requirement of each assigned flow. Eq.(4) denotes that the total link capacity, $C_{(u, t)}$ between switch $u$ and $t$ must be equal to or higher than demanding rate of total established $N_{u, t}$ links. Eq.(5) satisfies the delay requirements for each flow.

In C-RAN, we consider a cellular system based on OFDMA system where base station is located in the center of the cell. Let $\mathbb{D}=\{1,2, \ldots, D\}$ and $\mathbb{E}=$ $\{1,2, \ldots, E\}$ be the set of base stations and the set of users, respectively.

In OFDMA system, the total available bandwidth, $B_{w}$, is divided into $Q$ clusters and each cluster consists of a set of adjacent OFDM subcarriers to further reduce feedback load. $H_{d, e, q}$ is the channel coefficient between the $\mathrm{BS} d$ and the user $e$ that includes pathloss, 
shadowing and multipath components for the cluster $q$. The channel coefficient of a cluster is determined by $H_{d, e, q}=\bar{H}_{d, e, m}$ where $\bar{H}_{d, e, m}$ is channel coefficient of $m$ th subcarrier. The value of $m$ is determined by $m=(q-1) N_{Q}+\arg \min _{1 \leq i \leq N_{Q}}\left\{\left|\bar{H}_{d, e,(q-1) N_{q}+i}\right|^{2}\right\}$ for each cluster where $N_{Q}=M / Q$ is the number of subcarriers in one cluster and $M$ is the number of subcarriers. Let $\mathbb{Q}=\{1,2, \ldots, Q\}$ be the set of clusters.

Our aim is to satisfy data rate requirements of the users while maximizing the sum data rate for each base station during a whole transmission frame. The optimization problem for $\forall d \in \mathbb{D}$ is defined by

$$
\max \sum_{e=1}^{E} R_{d, e}
$$

subject to

$$
R_{d, e} \geqslant R_{d, e}^{t h} ; \quad \forall e \in \mathbb{E}
$$

where $R_{d, e}$ is data rate and $R_{d, e}^{t h}$ is the threshold data rate that must be satisfied at the end of the whole transmission frame for the user $e$ belonging to base station $d$.

The achievable data rate for each cluster is calculated by,

$$
R_{d, e, q}=\frac{B_{w}}{Q} \log _{2}\left(1+\frac{P_{d}\left|H_{d, e, q}\right|^{2}}{N_{0} B_{w}}\right)
$$

where $R_{d, e, q}$ is data rate for the cluster $q$, the user $e$ belonging to base station $d, P_{d}$ is transmitted power of base station $d, N_{0}$ is the noise power density.

\section{PRoposed JOINT ROUTING AND RESOURCE ALLOCATION ALGORITHM}

The proposed joint routing and resource allocation in SDMN is performed in two steps. In first step, the resource allocation is carried out in C-RAN to determine the $C_{f_{k}}^{w}$. In the second step, the proposed low complexity routing algorithm is executed in SDN controller to reduce power consumption.

In order to achieve these assignments, the SDN controller keeps track of the current topology and flow information with the support of C-RAN through the internal Flow Monitor application which communicates with the hardware using a south-bound Application Programming Interface (API) (usually OpenFlow or OFConfig). When a request for a new flow arrives to the SDN controller, it relays this request to the EnergyAware Routing application through a north-bound API on the orchestration plane. The routing application computes the necessary path using the constraints detailed above, updates its knowledge base with the new flow information and topology. Consequently, it commands the SDN controller via the Network Operating System (NOS) to apply the flows to the switches and then close or open ports and switches using the south-bound protocols.
In order to achieve Eq.(6) under the constraint Eq.(7) in OFDMA system, the resource allocation algorithm in Alg. 1 is performed to satisfy data rate requirements of the cellular users. At each transmission frame, the user having the best link quality is selected. When the user satisfies its data rate requirement, it is removed from the user set. Therefore, we provide a fair resource allocation to serve all users during the transmission.

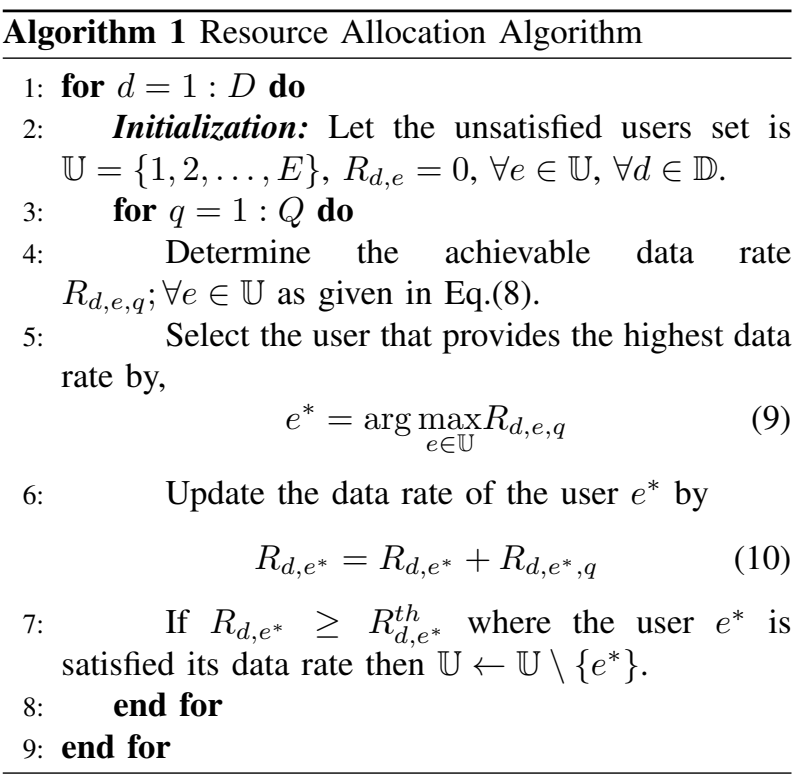

Then, we sum up all required data rate for each route having the same source and destination. Consequently, the calculated rate, $C_{f_{k}}^{w}$, corresponds to the link requirement of each flow:

$$
C_{f_{k}}^{w}=\sum_{g \in \mathbb{G}} \sum_{e=1}^{E} R_{g, e}
$$

where $\mathbb{G}$ consists of the base stations which are connected to the same OpenFlow switch.

The sets of input OpenFlow switches, II which are connected to the SDN controller and the output OpenFlow switches, $\mathbb{O}$, which are connected to base stations in the wireless networks are determined in a given network topology. Then, at the SDN controller, the proposed low complexity routing algorithm is performed as given in Alg. 2.

The problem of finding the routes of flows while minimizing the power requirements is in fact an NPcomplete problem whereas the incremental algorithm, in this paper, we propose a solution which has polynomial time complexity and is feasible to be run in real-time environments.

\section{Performance Results}

In this section, we give performance results of the proposed joint algorithm in terms of power consumption for various network topologies and traffic load. In each 


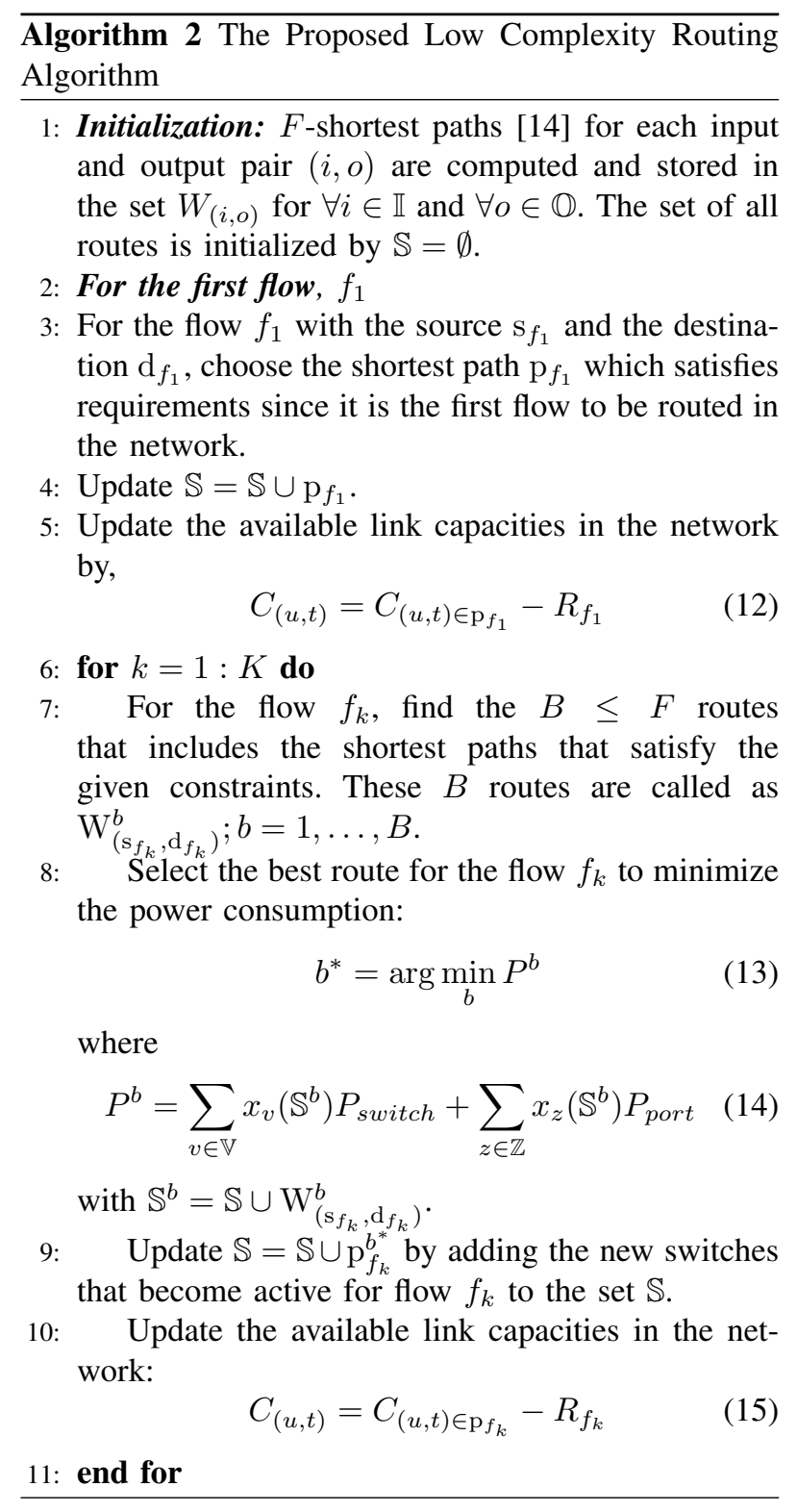

BS coverage, the number of connected users is generated according to Poisson distributions with the parameter $\lambda$. The traffic demands of these users are chosen uniformly among the values of $168 \mathrm{~kb} / \mathrm{s}, 336 \mathrm{~kb} / \mathrm{s}$ and $672 \mathrm{~kb} / \mathrm{s}$ for all network topologies. The consumed power of switches and ports are given in Table I.

TABLE I: The power used by network elements

\begin{tabular}{|c|c|}
\hline Power consumption per Switch & Power consumption per Port \\
\hline $80 \mathrm{~W}$ & $4 \mathrm{~W}$ \\
\hline
\end{tabular}

Firstly, we consider the network topology given in Figure 2. There are 20 OpenFlow switches with given total link capacities varying between $0.01 \mathrm{Gbps}, 0.02 \mathrm{Gbps}$, $0.05 \mathrm{Gbps}$ and $0.1 \mathrm{Gbps}$ depending on the predefined network architecture. For each OpenFlow switch, the number of total ports is calculated based on the capacity per port which is set as $10 \mathrm{Mbps}$.

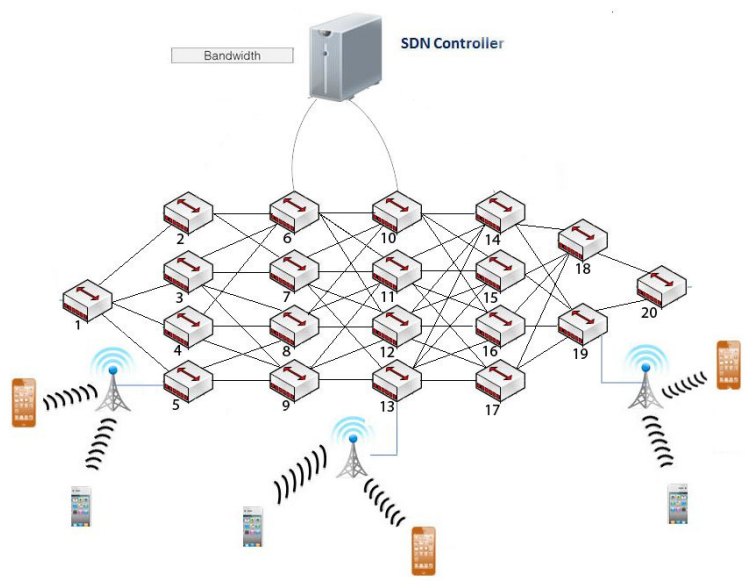

Fig. 2: First network topology

The performance of the proposed approach based on power saving for different users arrival rate is illustrated in Figure 3 for the first network topology. As a result of the proposed joint algorithm, the achievable percentage of power saving can reach up to $10 \%$ depending on the traffic load compared to the shortest path algorithm [14]. Because of the users which cannot achieve their data rate requirements due to their low SNR in wireless radio channel, the total demanded traffic for routing in SDMN is reduced, which leads to conservation of power in the network.

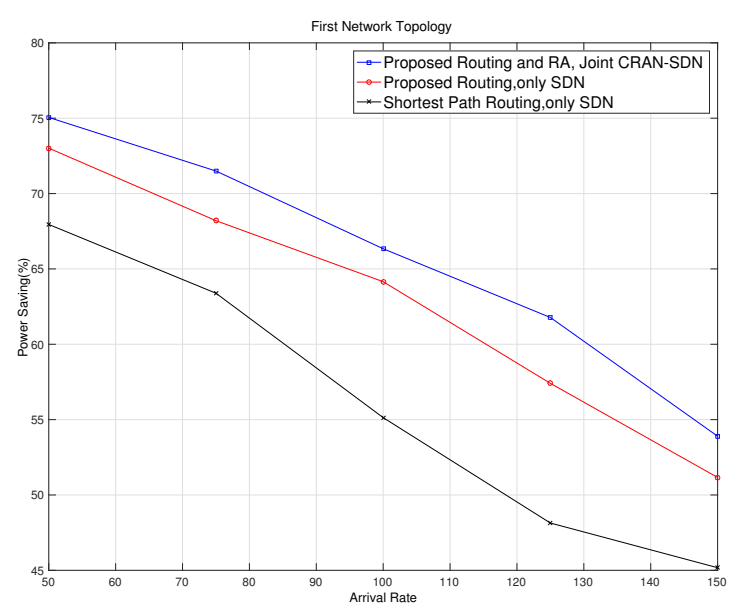

Fig. 3: Performance results of the proposed algorithm for the first network topology.

We also generate various topologies based on Waxman [15] to illustrate the performance of the proposed algorithm. In this topology, the network nodes are uniformly placed in the plane and edges defined according to distances between nodes. 
The probability to have an edge between nodes $u$ and $t$ is given by,

$$
\operatorname{Pr}(u, t)=\alpha e^{\frac{d^{l}}{\beta L}}
$$

where $\alpha$ determines the maximal link probability and $\beta$ controls the length of edges $d^{l}$ which is the distance between the node $u$ and $t$, and $L$ is the maximum distance between any two nodes. The parameters are given in Table II. In this model, all links between any two nodes have the capacity of $0.05 \mathrm{Gbps}$.

TABLE II: Parameters for generation of Waxman networks

\begin{tabular}{|c|c|c|c|}
\hline Topology & node count & $\alpha$ & $\beta$ \\
\hline First Waxman Network & 20 & 50 & 0.1 \\
\hline Second Waxman Network & 40 & 50 & 0.1 \\
\hline
\end{tabular}

In the first Waxman topology, three nodes are chosen as the possible sources and three nodes are selected as the possible destination. For the first Waxman topology, using the proposed joint algorithm, the power saving is increased up to $8 \%$ compared to the shortest path algorithm as given in Figure 4.

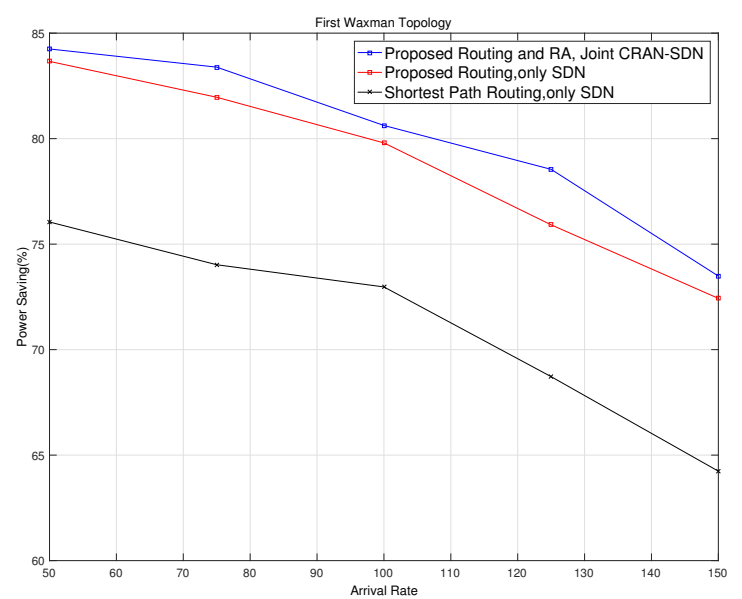

Fig. 4: Power saving for the first Waxman topology

In the second Waxman topology, three nodes are chosen as the possible sources and six nodes are selected as the possible destination. As given in Figure 5, the proposed joint algorithm achieves about up to $9 \%$ the power saving compared to shortest path algorithm for the second Waxman topology.

The proposed joint routing and resource allocation in SDMN achieves better performances for all types of network topologies compared to the proposed routing with only SDN in [6] which examined the energy efficient routing algorithm by decreasing the number of active switches while satisfying only throughput constraints without C-RAN.

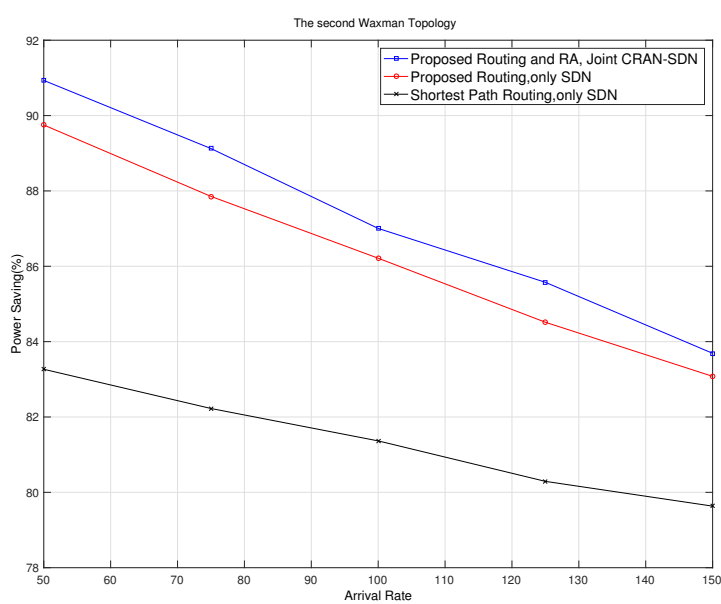

Fig. 5: Power saving for the second Waxman topology

The power consumption is calculated by taking into account both switch and port usage in total. According to the power consumption results given in Figure 6, the proposed joint routing and resource allocation in SDMN saves the power between $20 \mathrm{~W}$ and $100 \mathrm{~W}$ compared to the proposed routing with only SDN in [6] for all network topologies. Moreover, the power consumption the first network topology is less than the first Waxman topology since the number of OpenFlow swicthes in the first network topology are low.

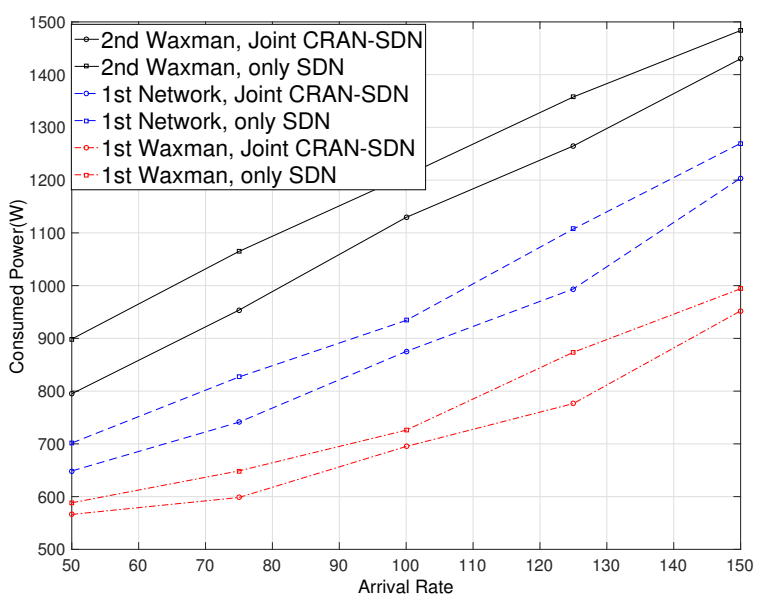

Fig. 6: Average power consumption for all network topologies

\section{CONCLUSIONS}

We have proposed an architecture including the joint routing and resource allocation to design power efficient SDMN by considering wireless channel conditions. Under low traffic load, the proposed approach enables to 
shut down the switches and the ports in the network while satisfying both throughput and delay requirements of the flows. We have illustrated the performance on power consumption of the proposed scheme in various network topologies and scenarios. As a future work, the proposed joint routing and resource allocation algorithm will be applied to the massive multiple input multiple output (MIMO) based heterogeneous systems.

\section{ACKNOWLEDGMENTS}

This work has been carried out in the framework of CELTIC-Plus project CP2012/2-5 SIGMONA.

\section{REFERENCES}

[1] S. Sezer, S. Scott-Hayward, P. Chouhan, B. Fraser, D. Lake, J. Finnegan, N. Viljoen, M. Miller, and N. Rao, Are we Ready for SDN? Implementation Challenges for Software-Defined Networks, IEEE Communications Magazine, vol. 51, no. 7, pp. 3643, 2013.

[2] S. Agarwal, M. Kodialam, and T. Lakshman, Traffic engineering in software defined networks, in IEEE Proceedings of IEEE 32th Annual Joint Conference of the IEEE Computer and Communications Societies (INFOCOM), pp. 2211-2219, April 2013.

[3] S. Oda, D. Nobayashi, Y. Fukuda and T. Ikenaga, Flow-based Routing Schemes for Minimizing Network Energy Consumption using OpenFlow, The Fourth International Conference on Smart Grids, Green Communications and IT Energy-aware Technologies, 2014.

[4] Markiewicz, A, Phuong Nga Tran, Timm-Giel, A., Energy consumption optimization for software defined networks considering dynamic traffic, IEEE 3rd International Conference on Cloud Networking (CloudNet),Luxembourg, October 2014.

[5] Ruiz-Rivera, A.; Kwan-Wu Chin; Soh, S., GreCo: An Energy Aware Controller Association Algorithm for Software Defined Networks, IEEE Communications Letters, vol.19, no.4, pp.541,544, April 2015.

[6] B. Ozbek, Y. Aydogmus, A. Ulas, B. Gorkemli, K. Ulusoy, Energy aware routing and traffic management for software defined networks, IEEE Conference on Network Softwarization (NetSoft), Seoul-Korean, 6-10 June 2016.

[7] $\mathrm{Yu} \mathrm{Y} \mathrm{S,} \mathrm{Ke} \mathrm{C} \mathrm{H.} \mathrm{Genetic} \mathrm{algorithm} \mathrm{based} \mathrm{routing} \mathrm{method} \mathrm{for}$ enhanced video delivery over software defined networks. Int $\mathrm{J}$ Commun Syst. 2018;31:e3391.

[8] H. Ali-Ahmad et al., CROWD: An SDN Approach for DenseNets, 2013 Second European Workshop on Software Defined Networks, Berlin, 2013, pp. 25-31.

[9] S. Auroux, M. Draxler, A. Morelli, et al., CROWD Dynamic Network Reconfiguration in Wireless DenseNets, in Proc. European Conference on Networks and Communications (EuCNC 2014).

[10] Savarese, G.; Vaser, M.; Ruggieri, M., A Software Defined Networking-based context-aware framework combining $4 G \mathrm{cel}$ lular networks with M2M, Wireless Personal Multimedia Communications (WPMC), 2013 16th International Symposium on , vol., no., pp.1-6, 24-27 June 2013.

[11] J. G. Andrews, S. Buzzi, W. Choi, S. Hanly, A. Lozano, A. C. Soong, and J. C. Zhang, What will $5 G$ be?, IEEE Journal on Selected Areas in Communications, Sep. 2014.

[12] Z.Guizani and N. Hamdi, C-RAN,H-RAN and F-RAN for 5G systems:Key capabilities and recent advances, Int. Journal of network Management, Volume 27, Issue 5, September/October 2017

[13] Wei, J., Yang, K., Zhang, G. et al., A QoS-Aware Joint Power and Subchannel Allocation Algorithm for Mobile Network Virtualization, in Wireless Pers Commun, https://doi.org/10.1007/s11277017-5053-y. Jan, 2019.

[14] J. Yen, Finding the $K$ shortest loopless paths in a network, Theory Series, vol. 17, no. 11, pp. 712 - 716, 1971.

[15] B. Waxman, Routing of Multipoint Connections, IEEE J. Select. Areas Commun., December 1988. 